\title{
Determination of the Relationship Between Kobayashi, Sano, and Egami Criteria and Prevalence of Intravenous Immunoglobulin Resistance and Coronary Artery Aneurysm in Iranian Children with Kawasaki Disease
}

This article was published in the following Dove Press journal: Open Access Rheumatology: Research and Reviews

\author{
Niloufar Shashaani ${ }^{1}$ \\ Reza Shiari (iD ${ }^{2}$ \\ Abdullah Karimi ${ }^{3}$ \\ Shima Salehi iD ${ }^{4}$ \\ Roxana Ghanaei ${ }^{3}$ \\ Mehrnoush Hassas Yeganeh (iD) ${ }^{2}$ \\ Sara Shiari' \\ Khosro Rahmani iD ${ }^{2}$ \\ Vadood Javadi Parvaneh (iD ${ }^{2}$ \\ 'Faculty of Medicine, Shahid Beheshti \\ University of Medical Sciences, Tehran, \\ Iran; ${ }^{2}$ Department of Pediatric \\ Rheumatology, Mofid Children's Hospital, \\ Shahid Beheshti University of Medical \\ Sciences, Tehran, Iran; ${ }^{3}$ Department of \\ Infectious Diseases, Mofid Children's \\ Hospital, Shahid Beheshti University of \\ Medical Sciences, Tehran, Iran; ${ }^{4}$ Faculty of \\ Medicine, Ali Asghar Children's Hospital, \\ Iran University of Medical Sciences, \\ Tehran, Iran
}

Introduction: Kawasaki disease $(\mathrm{KD})$ is a systemic vasculitis that occurs mostly in children under five years old. Kawasaki affects the middle-size arteries, especially the coronary arteries. Therefore, without adequate treatment, it may cause coronary artery aneurysm in $25 \%$ of patients. The purpose of this study was to investigate the relationship between Kobayashi, Sano, and Egami criterions with coronary artery aneurysm in KD patients during the last ten years and to identify risk factors in patients with intravenous immunoglobulin (IVIG)-resistant and coronary artery aneurysms.

Methodology: Medical records of 363 Kawasaki patients referred during 2008-2017 were reviewed. Patients' demographic data and Kobayashi, Sano, and Egami scores of each patient were calculated. Based on echocardiographic findings, cases of coronary artery aneurysm were determined. Sensitivity, specificity, positive and negative predictive value, and the accuracy of each criterion were determined to predicting IVIG resistance and detect coronary artery aneurysm.

Results: There was a slight relationship between IVIG-resistance in Kawasaki children and its prediction based on the Kobayashi risk score, but no relationship was found between the Egami and Sano criteria. Sixty-three patients (17.4\%) had coronary artery lesions (CALs) on time of diagnosis. There were no statistically significant differences between gender and mean age of children with and without CALs. Also, there was no significant relationship between coronary artery aneurysm in Kawasaki children and its prediction based on the above three risk factors. The area under the ROC-curve of all three risk measures of Kobayashi, Egami, and Sano indicated that all three criteria were not useful in predicting CALs.

Conclusion: Despite the low accuracy of the three above criteria to predictive of patients with IVIG resistance, it seems that the variables of age, duration of fever, and C-reactive protein (CRP) are more useful than other variables and may be utilized to evaluate patients by establishing a more appropriate cut-off point.

Keywords: Kawasaki disease, coronary artery aneurysm, IVIG-resistant, Kobayashi, Sano, Egami

\section{Introduction}

Kawasaki disease (KD) is an acute systemic vasculitis that mostly occurs in infants and children, especially in Asian children. ${ }^{1}$ The clinical manifestations include fever, rash, conjunctivitis, cervical lymphadenitis, inflammation of the mouth and
Correspondence: Reza Shiari

Tel +98-2I-22227033

Email shiareza@yahoo.com
Open Access Rheumatology: Research and Reviews 2020:12 187-192 
lips, erythema, and edema of the hands and legs. ${ }^{2}$ Although the exact cause of KD is not yet understood, it seems that it is the pathophysiological response to endothelial cell activation that leads to stimulation, incidence, and overproduction of cytokines, chemokines, growth factors, and other vasoactive substances. ${ }^{3,4}$

Severe systemic inflammation and vasculitis can cause cardiovascular involvement, especially coronary artery involvements, including spasm or aneurysm. Coronary artery lesions (CALs) occur in $15-25 \%$ of the KD children and may lead to myocardial infarction, sudden death, or late-onset ischemic heart disease, which is the leading cause of long-term mortality. ${ }^{5}$ Therefore, the ultimate goal of treating acute KD is to reduce systemic inflammation and vasculitis as quickly as possible and consequently prevent the progression of coronary artery injury and thrombosis. ${ }^{6}$

It seems that the leading cause of acute coronary artery aneurysm is intravenous immunoglobulin (IVIG) resistance. About $15-20 \%$ of the patients with acute Kawasaki have been reported to be IVIG-resistant even with standard treatment using high IVIG doses. Patients with IVIG-resistant KD are at higher risk for CALs. ${ }^{7}$ Recent studies on the identification and prediction of IVIG resistance have been focused on the development of therapeutic alternatives in the early stage of the disease and the prevention of coronary artery lesions.

To assess the risk factors for predicting IVIG resistance and consequently for diagnosing of CALs in Japanese children with KD, several criteria such as those introduced by Kobayashi, Sano, and Egami that are mostly used methods for clinical assessment of this disease in Japan. ${ }^{8-10}$ Therefore, predicting the prognosis of and knowing the risk factors for $\mathrm{KD}$ will make it possible to recommend more accurate control and prevention methods for this group of patients in the Iranian population.

Since no study on the sensitivity and specificity of these criteria in predicting of IVIG resistance and CALs has been conducted in Iran so far, the present study was designed to determine the relationship between Kobayashi, Sano and Egami criteria and prevalence of IVIG resistance and coronary artery aneurysm in Iranian children with KD.

\section{Patients and Methods}

\section{Patients}

The current study was approved by the ethics committee of Shahid Beheshti University of Medical Sciences. Medical records included demographics, clinical manifestations, laboratory data, and the treatment of all children who presented with KD to two pediatric tertiary medical centers in Iran between 2008 and December 2016 were reviewed. The inclusion criteria for this study were based on a clinical diagnosis of $\mathrm{KD}$ which defined as fever for more than five days and at least four of the five KD criteria. Admission during the acute period of the illness and treatment with at least one IVIG course $(2 \mathrm{~g} / \mathrm{kg})$ plus aspirin were also included in the inclusion criteria. Patients who were admitted during the sub-acute phase excluded from the study.

Patients also underwent echocardiography at the time of admission, and coronary artery aneurysms (CAA) were determined. We described CAA as the left anterior descending (LAD) and/or the right coronary artery (RCA) zscore $>3$ or original CAA criteria of the Japanese Ministry of Health and Welfare in CA segments for which z-scores are not available (CA dimension $>3 \mathrm{~mm}$ for patients $<5$ years of age and $>4 \mathrm{~mm}$ in patients $\geq 5$ years of age). ${ }^{5}$ Left main CA (LMCA) z-score was not used for inclusion due to previously reported variability in LMCA anatomy and measurement. $^{5}$

All data required to assess each of the Kobayashi, Sano, and Egami criteria for the diagnosis of coronary artery aneurysm, such as CRP at the beginning of admission, were determined and the scores of each criterion were calculated. Then, based on each of the above risk criteria, resistance to IVIG was determined. In this study, IVIG resistance was indicated by a persistent fever after the first dose of IVIG, which was defined as having a fever after 24-72 hours.

\section{Statistical Analysis}

Data were analyzed with SPSS software and MedCalc version 12.7.1.0. To analyze the continuous data, we used the Student's $t$-test for normally distributed variables and Kruskal-Wallis for non-parametric variables. One-way analysis of variance (ANOVA) was used to compare multiple group averages. A linear logistic regression analysis was performed in an attempt to determine predictors for IVIG resistance. In all the analyses, a two-sided p-value of $<0.05$ was considered statistically significant. Means were accompanied by standard deviations and medians by ranges.

\section{Results}

The study comprised 363 Iranian patients (59.8\% male), and their mean age was $4.3 \pm 2.9$ years. The median time 
Table I Comparison of the Kobayashi, Egami, and Sano Criterions Between IVIG-Responsive and IVIG Resistant Patients

\begin{tabular}{|c|c|c|c|c|c|}
\hline \multicolumn{2}{|l|}{ Parameter } & \multirow{8}{*}{$\begin{array}{l}\text { IVIG Responsive } \mathbf{n}= \\
276 \\
64(23.2 \%) \\
39(14.1 \%) \\
24(8.7 \%) \\
102(37 \%) \\
237(85.9 \%) \\
42(15.2 \%) \\
105(38 \%)\end{array}$} & \multirow{8}{*}{$\begin{array}{l}\text { IVIG Resistant } \mathbf{n}= \\
87 \\
29(33.3 \%) \\
27(31.0 \%) \\
9(10.3 \%) \\
30(34.5 \%) \\
67(77.0 \%) \\
29(33.3 \%) \\
28(32.2 \%)\end{array}$} & \multirow{8}{*}{$\begin{array}{l}\text { P-value } \\
0.067 \\
0.001^{*} \\
0.670 \\
0.703 \\
0.066 \\
0.001^{*} \\
0.372\end{array}$} & \multirow{8}{*}{$\begin{array}{l}\text { Odds Ratio (95\% } \\
\text { CI) } \\
1.66(0.98-2.80) \\
2.74(I .55-4.82) \\
1.21(0.54-2.72) \\
0.90(0.54-1.49) \\
0.55(0.30-1.01) \\
2.79(1.60-4.85) \\
0.77(0.46-1.29)\end{array}$} \\
\hline Kobayashi Risk & Sodium ( $\leq 133 \mathrm{mmol} / \mathrm{L})$ & & & & \\
\hline Factor & Fever ( $\leq 4$ days) & & & & \\
\hline & AST ( $\geq 100 \mathrm{IU} / \mathrm{L})$ & & & & \\
\hline & Neutrophils ( $\geq 80 \%$ ) & & & & \\
\hline & CRP $(\geq 10 \mathrm{mg} / \mathrm{dL})$ & & & & \\
\hline & Age ( $\leq 12$ months) & & & & \\
\hline & Platelets $\left(\leq 300 \times 10^{9} / \mathrm{L}\right)$ & & & & \\
\hline \multirow[t]{5}{*}{ Egami Risk Factor } & Age (< 6 months) & 25 (9.1\%) & 13 (I4.9\%) & 0.158 & $1.76(0.86-3.62)$ \\
\hline & Fever ( $<4$ days $)$ & 40 (I4.5\%) & $24(27.6 \%)$ & $0.009 *$ & $2.25(1.26-4.00)$ \\
\hline & Platelets $(\leq 300 \times 109 / \mathrm{L})$ & 101 (36.6\%) & 26 (29.9\%) & 0.303 & $0.74(0.44-1.24)$ \\
\hline & $\mathrm{CRP}(\geq 8 \mathrm{mg} / \mathrm{dL})$ & $235(85.1 \%)$ & $72(72.4 \%)$ & $0.010 *$ & $0.46(0.26-0.8 \mathrm{I})$ \\
\hline & ALT ( $\geq 80 \mathrm{IU} / \mathrm{L})$ & 45 (16.3\%) & $14(16.1 \%)$ & 1.00 & $0.98(0.51-1.90)$ \\
\hline \multirow[t]{3}{*}{ Sano Risk Factor } & CRP $(\geq 8.0 \mathrm{mg} / \mathrm{dL})$ & $232(84.1 \%)$ & $64(73.6 \%)$ & $0.038^{*}$ & $0.53(0.30-0.94)$ \\
\hline & $\begin{array}{l}\text { Total Bilirubin }(\geq 0.9 \mathrm{mg} / \\
\mathrm{dL})\end{array}$ & $9(3.3 \%)$ & $5(5.7 \%)$ & 0.580 & I.8I (0.59-5.6) \\
\hline & AST ( $\geq 200 \mathrm{IU} / \mathrm{L})$ & 15 (5.4\%) & $3(3.4 \%)$ & 0.580 & I. $(0.18-2.2)$ \\
\hline
\end{tabular}

Note: *Statically significant (P-value<0.05).

Abbreviations: ALT, alanine transaminase; AST, aspartate transaminase; CRP, C-reactive protein; IVIG, intravenous immunoglobulin.

from fever onset until the first treatment was $7.4 \pm 2.5$ days. All patients were treated with $2 \mathrm{~g} / \mathrm{kg}$ of intravenous immunoglobulin, and there were 87 (24.0\%) IVIG resistant patients. There was a significant difference between the responder and resistant group in age, duration of fever, and C-reactive protein (CRP) level in comparing Kobayashi, Egami, and Sano criteria (Table 1).

C-reactive protein on admission, with an odds ratio of $0.53(\mathrm{p}=0.038)$, Age of less than 1 year (odds ratio 0.736, $\mathrm{p}=0.005$ ) and fever of less than four days (odds ratio $0.733, \mathrm{p}=0.037$ ) were the only variables that remained significant (Table 2 and Figure 1). However, these variables contribute to the final R2 (21.8) was relatively low. Each of the three scores; Kobayashi, Sano, and Egami, demonstrated low sensitivity $(8.1-48.3 \%)$, positive predictive value (29.2-31.7), and negative predictive value

Table 2 Multivariate Analysis, Using Binary Logistic Regression Model with Log It Link Function and Stepwise Selection for Evaluating the Predisposing Factors for the Occurrence of Resistance to Intravenous Immunoglobulin

\begin{tabular}{|l|l|l|l|l|}
\hline Parameter & $\begin{array}{l}\text { Probability } \\
\text { Ratio }\end{array}$ & P-value & $\mathbf{X}^{\mathbf{2}}$ & P-value \\
\hline Fever ( $\leq 4$ days $)$ & 0.736 & 0.005 & 21.86 & $<0.001$ \\
Age $(\leq 12 \mathrm{months})$ & 0.733 & 0.037 & & \\
CRP $(\geq 8 \mathrm{mg} / \mathrm{dL})$ & - & 0.111 & - & - \\
\hline
\end{tabular}

Abbreviation: CRP, C-reactive protein.
(76.4-80.4). The Kobayashi and Egami scores demonstrated a moderate specificity (66.3-80.4\%), while the Sano score demonstrated a good specificity (93.8\%).

Of the 363 patients, 63 developed CALs on disease onset. There was no significant difference between the age and gender of patients with and without CALs $(\mathrm{P}>0.05)$. Also, there was no significant difference between Kobayashi, Egami, and Sano scores in patients with and without CALs $(\mathrm{P}>0.05)$. Sensitivity, specificity, positive predictability, negative predictability, and accuracy of three scoring systems for the diagnosis of CALs are shown in Table 3.

\section{Discussion}

Kawasaki disease is believed to be the commonest vasculitis of medium vessels in Asian children. However, many children with KD remain undiagnosed possibly because of the lack of cognizance amongst pediatricians. The development of coronary artery abnormalities is the symbol of $\mathrm{KD}$ and accounts for most morbidity and mortality associated with the disease. ${ }^{1}$ This two-center observational study retrospectively evaluated the applicability of three Japanese scoring systems, Kobayashi, Egami, and Sano that assess the risk of IVIG resistance in the Iranian population with Kawasaki disease.

Based on the results of our study, it can be stated that these three criteria are more specific than sensitive in 


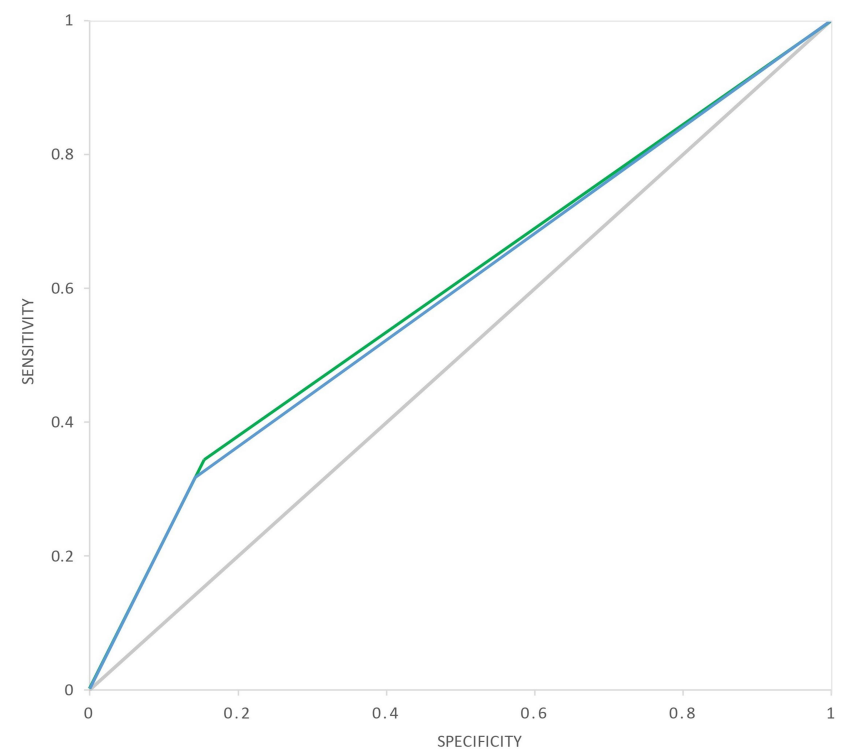

Figure I Univariate analysis and area under the ROC curve for fever $\leq 4$ days and age $\leq$ I year, in predicting IVIG resistance in children with Kawasaki disease. (Blue line: Fever $\leq 4$ days; Green line: Age $\geq 12$ months; Grey line: Reference line; Diagonal segments are produced by ties).

Abbreviations: IVIG, intravenous immunoglobulin; ROC, receiver operating characteristics.

predicting IVIG resistance and coronary artery involvement in Kawasaki patients, suggesting that correction is needed. Accordingly, identifying non-Japanese patients at risk for IVIG resistance remains a challenge. Therefore, it is necessary to develop a new risk assessment tool to predict IVIG resistance in children with Kawasaki disease in future treatment strategies.

There are many differences in the epidemiology of KD among the Japanese population compared to the non-Asian population. The risk of the disease is high and is even greater among siblings affected in Japan. ${ }^{11}$

In our study population, the mean age of patients was 4.3 years, and the prevalence of disease was higher in males $(59.8 \%)$. These statistics were consistent with data from patients with KD in many ethnic groups. ${ }^{12}$ However, the highest incidence of the disease in Japanese patients was between 6 and 11 months. ${ }^{13}$
The sensitivity of age, duration of fever, and CRP level for predicting resistance to IVIG treatment in the current study were low (8-48\%), and specificity was in the moderate range (66-93\%).

In our population, Japanese scoring methods were able to identify most low-risk patients; however, these scoring systems were unable to identify high-risk patients who need more care and could benefit from early and aggressive treatment intervention.

Our results showed that, for the Iranian patient, the scoring methods derived from studies of Japanese patients are a poor predictor of resistance to IVIG treatment. Our findings are also consistent with other studies on nonAsian populations. ${ }^{14,15}$

We attempted to develop a predictive model to identify IVIG treatment resistance risk factors in our study group. In the study by Sleeper et al, male gender and low serum albumin levels founded as two independent risk factors for IVIG resistance, however, none of these criteria were included in the Japanese criterion score. ${ }^{12}$

A study by Park et al showed that the mean alanine transaminase, total bilirubin, aspartate aminotransferase (AST), neutrophil percent, and NT-proBNP levels were significantly higher in IVIG resistant patients and the levels of sodium and HDL were significantly lower in those patients. Their study also found no differences in risk factors such as CRP, platelet count, and albumin. They concluded that, based on multivariate logistic regression analysis, two serum levels of ALT and bilirubin were identified as independent predictors of IVIG resistance. ${ }^{16}$

In the present study, we identified age less than one year, and fever of 4 days or less as independent risk factors for IVIG resistance. However, these factors had a low predictive value.

According to Our findings in echocardiography, 63 patients $(17.4 \%)$ had coronary artery aneurysms on time of diagnosis. There was no statistically significant difference between gender and mean age of children with KD with and without CALs.

Table 3 Sensitivity, Specificity, Positive Predictability Value, Negative Predictability Value, and Accuracy of Kobayashi, Egami, and Sano Criterions to Diagnosis of Coronary Artery Aneurysms

\begin{tabular}{|l|l|l|l|l|l|}
\hline Risk Scoring System & Sensitivity & Specificity & PPV & NPV & Accuracy \\
\hline Kobayashi $\geq 4$ & $34.9 \%$ & $62.3 \%$ & $16.3 \%$ & $82.0 \%$ & $57.6 \%$ \\
Egami $\geq 3$ & $28.6 \%$ & $79.7 \%$ & $22.8 \%$ & $84.2 \%$ & $70.8 \%$ \\
Sano $\geq 2$ & $6.4 \%$ & $93.3 \%$ & $16.7 \%$ & $82.6 \%$ & $78.2 \%$ \\
\hline
\end{tabular}

Abbreviations: NPV, negative predictability value; PPV, positive predictability value. 
Also, our results showed that three Japanese scoring systems were unable to predict coronary artery aneurysm in Iranian patients with Kawasaki disease. The sensitivity and specificity of these three scoring systems in predicting coronary artery aneurysm were (34-6\%) and (93-82\%), respectively. Also, the ROC-Curve level of Kobayashi, Egami, and Sano in diagnosing of the coronary aneurysm was $48.6 \%$, $54.1 \%, 49.8 \%$, respectively, that indicating all three scoring systems are not statistically useful in predicting CALs.

Similar results were also found in other studies of nonJapanese patients. In the study by Davies et al, coronary artery aneurysm occurred in $33.9 \%$ of patients, and there was no significant difference between patients with and without IVIG in coronary artery involvement. In their study, the sensitivity and specificity of the Kobayashi scoring system for predicting coronary artery aneurysm were $60 \%$ and $36 \%$, respectively. ${ }^{14}$

Due to the high prevalence in the Japanese population, many studies have concluded that genetics is a reliable and relevant factor in the onset of the disease. It is also more likely to occur among siblings and children whose parents and family members have the condition $\mathrm{KD}{ }^{6}{ }^{6}$ These findings are a possible explanation for the question of why Japanese scoring practices performed poorly in our research and other populations, thus making the individual assessment of each ethnic group critical.

\section{Limitations}

Although this study was the first comprehensive study in the Iranian pediatric community with acceptable sample size, there were some limitations. Lack of one pediatric cardiologist for echocardiography studied, and lack of some data such as ferritin of serum were the main limitations of our study.

\section{Conclusion}

Kobayashi, Egami, and Sano risk scores were not useful for predicting coronary artery involvement and IVIG resistance in Kawasaki patients. However, despite the strength of the above criteria in correctly diagnosing patients with IVIG resistance, the variables of CRP, age, and duration of fever appear to perform more useful than other variables and may be more appropriate for assessing patients by establishing criteria. Therefore, the need to develop a new risk assessment tool to predict IVIG resistance in children with Kawasaki disease still seems to be necessary.

\section{Abbreviations}

ALT, alanine transaminase; ANOVA, one-way analysis of variance; AST, aspartate aminotransferase; CALs, coronary artery lesions; CRP, C-reactive protein; IVIG, intravenous immunoglobulin; KD, Kawasaki disease.

\section{Data Sharing Statement}

The datasets analyzed during the current study available from the corresponding author on reasonable request.

\section{Ethics Approval and Consent to Participate}

Study protocol approved by Shahid Beheshti University of Medical Sciences ethic committee and all patients (or their parents) have been informed and gave their written consent before starting the study.

\section{Funding}

There is no funding to report.

\section{Disclosure}

The authors declare that they have no competing interests for this work.

\section{References}

1. Shiari R. Kawasaki disease; A review article. Arch Pediatr Infect Dis. 2014;2(1):154-159.

2. Kawasaki TJA. Acute febrile mucocutaneous syndrome with lymphoid involvement with specific desquamation of the fingers and toes in children. Arerugi. 1967;16:178-222.

3. Newburger JW, Takahashi M, Gerber MA, et al. Diagnosis, treatment, and long-term management of Kawasaki disease: a statement for health professionals from the committee on Rheumatic Fever, Endocarditis and Kawasaki Disease, council on Cardiovascular Disease in the young, American Heart Association. Circulation. 2004;110(17):2747-2771.

4. Dimitriades VR, Brown AG, Gedalia A. Kawasaki disease: pathophysiology, clinical manifestations, and management. Curr Rheumatol Rep. 2014;16(6):423.

5. Friedman KG, Gauvreau K, Hamaoka-Okamoto A, et al. Coronary artery aneurysms in Kawasaki disease: risk factors for progressive disease and adverse cardiac events in the US population. J Am Heart Assoc. 2016;5(9):e003289.

6. McCrindle BW, Rowley AH, Newburger JW, et al. Diagnosis, treatment, and long-term management of Kawasaki disease: a scientific statement for health professionals from the American Heart Association. Circulation. 2017;135(17):e927-e99.

7. Dionne A, Le C-K, Poupart S, et al. Profile of resistance to IVIG treatment in patients with Kawasaki disease and concomitant infection. PLoS One. 2018;13(10):e0206001.

8. Sano T, Kurotobi S, Matsuzaki K, et al. Prediction of non-responsiveness to standard high-dose gamma-globulin therapy in patients with acute Kawasaki disease before starting initial treatment. Eur J Pediatr. 2007;166(2):131-137. 
9. Egami K, Muta H, Ishii M, et al. Prediction of resistance to intravenous immunoglobulin treatment in patients with Kawasaki disease. J Pediatr. 2006;149(2):237-240.

10. Kobayashi T, Inoue Y, Takeuchi K, et al. Prediction of intravenous immunoglobulin unresponsiveness in patients with Kawasaki disease. Circulation. 2006;113(22):2606-2612.

11. Hirata S, Nakamura Y, Yanagawa H. Incidence rate of recurrent Kawasaki disease and related risk factors: from the results of nationwide surveys of Kawasaki disease in Japan. Acta Paediatr. 2001;90 (1):40-44.

12. Sleeper LA, Minich LL, McCrindle BM, et al. Evaluation of Kawasaki disease risk-scoring systems for intravenous immunoglobulin resistance. $J$ Pediatr. 2011;158(5):831-835.
13. Nakamura Y, Yashiro M, Uehara R, et al. Epidemiologic features of Kawasaki disease in Japan: results of the 2009-2010 nationwide survey. J Epidemiol. 2012;1203070303.

14. Davies S, Sutton N, Blackstock S, et al. Predicting IVIG resistance in UK Kawasaki disease. Arch Dis Child. 2015;100(4):366-368.

15. Saundankar J, Yim D, Itotoh B, et al. The epidemiology and clinical features of Kawasaki disease in Australia. Pediatrics. 2014;133(4): e1009-e14.

16. Park HM, Lee DW, Hyun MC, Lee S. Predictors of nonresponse to intravenous immunoglobulin therapy in Kawasaki disease. Korean J Pediatr. 2013;56(2):75.

\section{Publish your work in this journal}

Open Access Rheumatology Research and Reviews is an international, peer-reviewed, open access journal publishing original research, reports, editorials, reviews and commentaries on all aspects of clinical and experimental rheumatology in the clinic and laboratory including the following topics: Pathology, pathophysiology of rheumatological diseases; Investigation, treatment and management of rheumatological diseases; Clinical trials and novel pharmacological approaches for the treatment of rheumatological disorders. The manuscript management system is completely online and includes a very quick and fair peer-review system, which is all easy to use. Visit http://www.dovepress.com/testimonials.php to read real quotes from published authors. 